\title{
Broadband Transient Monitor using the Swift/BAT and the MAXI/GSC data
}

\section{T. Sakamoto*, Y. Oda and A. Yoshida}

Aoyama Gakuin University, Japan

E-mail: tsakamotodphys.aoyama.ac.jp

\begin{abstract}
"Time-domain astronomy" is one of the frontier field of astronomy for the next decade. Since the most of the transient sources show the temporal variation in a broad energy range, it would be ideal to have the real-time broadband transient monitor. We are constructing the real-time broadband transient monitor combining the Swift/BAT and the MAXI/GSC data which can cover from $2 \mathrm{keV}$ to $200 \mathrm{keV}$ without any gap. Therefore, our transient monitor covers the dynamic range of two order of magnitudes. Our monitor will be available to the public. We show the gallery of the light curves for selected sources based on our transient monitor process and discuss about the future plan of our project.
\end{abstract}

Swift: 10 Years of Discovery,

2-5 December 2014

La Sapienza University, Rome, Italy

\footnotetext{
* Speaker.
} 


\section{Introduction}

Most of the high energy astrophysical sources show a temporal variability in a broad spectral range. The Burst Alert Telescope (BAT; [ []]) onboard Swift spacecraft [] has been monitoring the hard X-ray sky (15-200 keV) since 2004. On the other hand, Gas Slit Camera (GSC; [3] ) onboard MAXI platform [䧃] on the International Space Station has been scanning the soft X-ray sky (2-20 $\mathrm{keV}$ ) since 2009. Although both instrument teams provide the real-time light curve data of their individual instruments, there is no service combining the both data and making the board-band light curves of high energy sources available to the public. Our goal is to construct the real-time broadband transient monitor combing the Swift/BAT and the MAXI/GSC data.

\section{Analysis}

First, we created the mirror of the Swift BAT data from HEASARC ${ }^{1}$ to our server. Since it takes a significant amount of time to download the BAT data from U.S. (HEASARC) to Japan, this first process is crucial to process the data in a timely fashion. Currently, one of our pipeline is continuously copying the updated data from HEASARC and also from the Swift Quick-Look site ${ }^{2}$. Next, we processed the Swift BAT survey data from August 2009 (when the MAXI GSC started to collect the science data) up to August 2014 using the standard batsurvey script. The process was performed in the standard eight energy bands (14-20 keV, $20-24 \mathrm{keV}, 24-35 \mathrm{keV}, 35-50 \mathrm{keV}$, 50-75 keV, 75-100 keV, 100-150 keV, and 150-195 keV) with the finest time sample ( 300 sec; called DPH option in the script) and including 146 bright hard X-ray sources in the input catalog. All the generated output catalogs were merged using bat survey-catnum script for individual source basis. Then, we applied the off-axis correction [] based on the Crab nebula data collected on 2004-2005 to the merged catalog files. The initial BAT eight energy bands were combined into four energy bands (14-24 keV, 24-50 keV, 50-100 keV and 100-195 keV), and also, the original light curve rates were binned to daily-averaged rates using rebingausslc for the most of the sources. The publically available MAXI light curves in three energy bands (2-4 keV, 4-10 keV and $10-20 \mathrm{keV}$ ) were downloaded from the public web page at Riken ${ }^{3}$.

\section{Gallery of the Swift/BAT and the MAXI/GSC Broad-band Light curves}

Figure $\square-\$$ show the gallery of the Swift/BAT and the MAXI/GSC broad-band light curves for selected bright sources. The data and the energy bands of the light curves in each panel are the MAXI/GSC 2-4 keV, the MAXI/GSC 4-10 keV, the MAXI/GSC 10-20 keV, the Swift/BAT 14-24 $\mathrm{keV}$, the Swift/BAT 24-50 keV, the Swift/BAT 50-100 keV and the Swift/BAT 100-195 keV from top to bottom panel.

Continuous variabilities in a few days to a few tens of days time scale up to $50 \mathrm{keV}$ are clearly seen in Vela X-1 (Figure W), SMC X-1 (Figure [1) and LMC X-4 (Figure W). The soft/hard

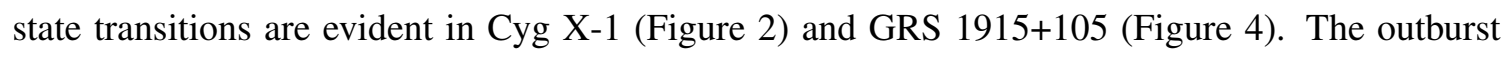

\footnotetext{
${ }^{1}$ http://heasarc.gsfc.nasa.gov/FTP/swift/data/obs/

${ }^{2}$ http://swift.gsfc.nasa.gov/cgi-bin/sdc/ql?

${ }^{3}$ http://maxi.riken.jp/top/index.php?cid=1\&disp_mode=curves
} 
episodes of A0535+262 are clearly visible up to $100 \mathrm{keV}$ (Figure [1). As seen in those examples, our light curve will give us a very broad view of high energy sources both from temporal and spectral properties.

\section{Future}

As seen in the figures, the data gaps in the Swift/BAT light curves are seen. We are currently identifying the missing data and processing those data to fill the gaps. We are also updating our data downloading pipeline not to miss any updated data. The automatic pipeline to analyze the Swift/BAT survey data in real-time is needed to be installed not to miss the interesting transient events for monitoring high energy sources. We are planning to make our product available to the public sometime on 2015 .

\section{References}

[1] S. D. Barthelmy et al., The Burst Alert Telescope (BAT) on the Swift Midex Mission, Space Sci. Rev., 120, (2005) 143

[2] N. Gehrels et al., The Swift Gamma-Ray Burst Mission, ApJ, 611, (2004) 1005

[3] T. Mihara et al., Gas Slit Camera (GSC) onboard MAXI on ISS, PASJ, 63, (2011) S623

[4] M. Matsuoka et al., The MAXI Mission on the ISS: Science and Instruments for Monitoring All Sky X-Ray Images, PASJ, 61, (2009) 999

[5] J. Tueller et al., The 22 Month Swift-BAT All-Sky Hard X-ray Survey, ApJS, 186, (2010) 378 


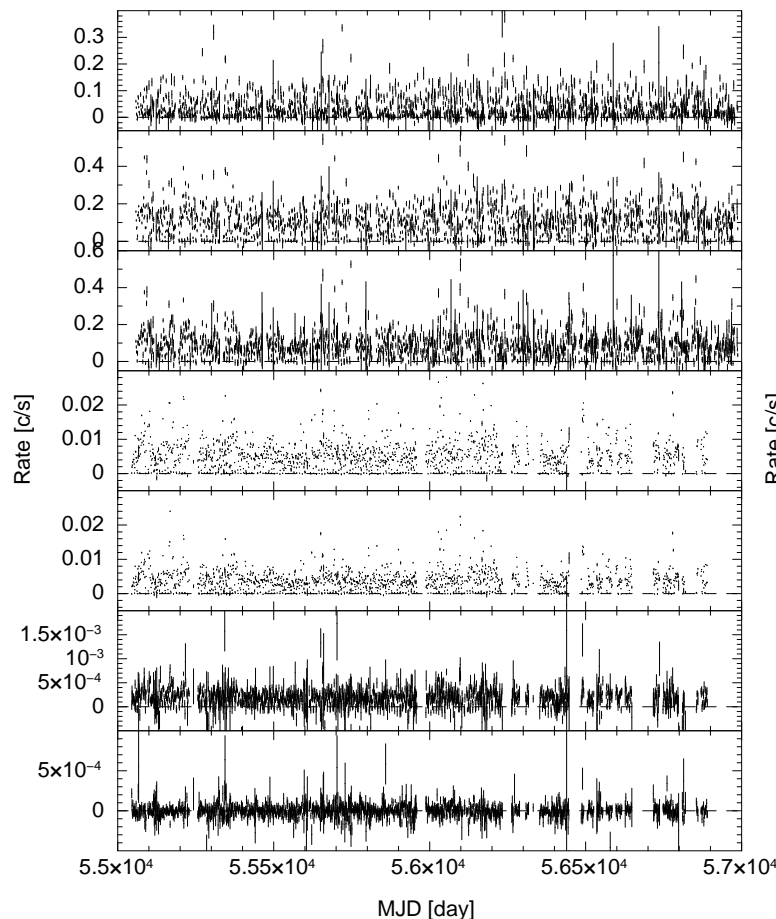

Figure 1: Vela X-1

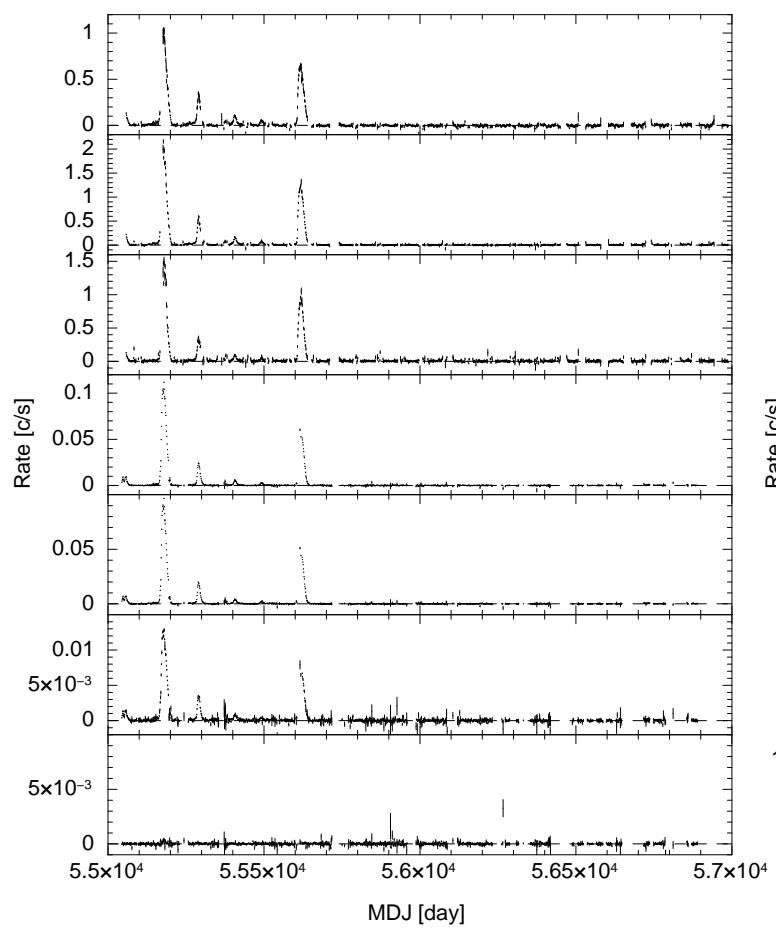

Figure 3: A0535+262

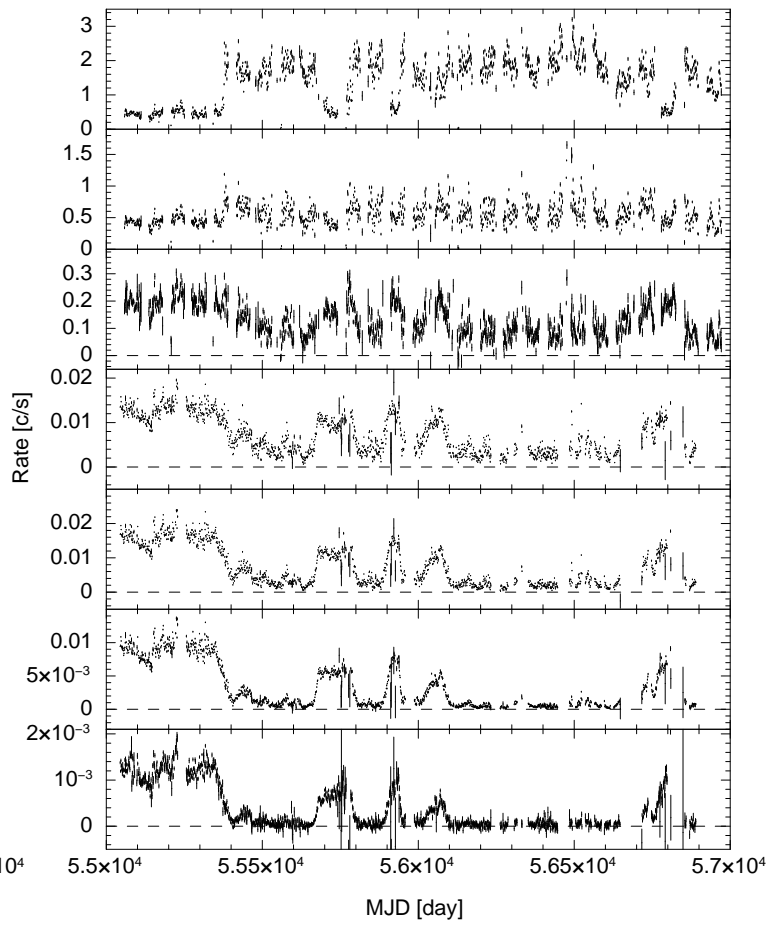

Figure 2: Cyg X-1

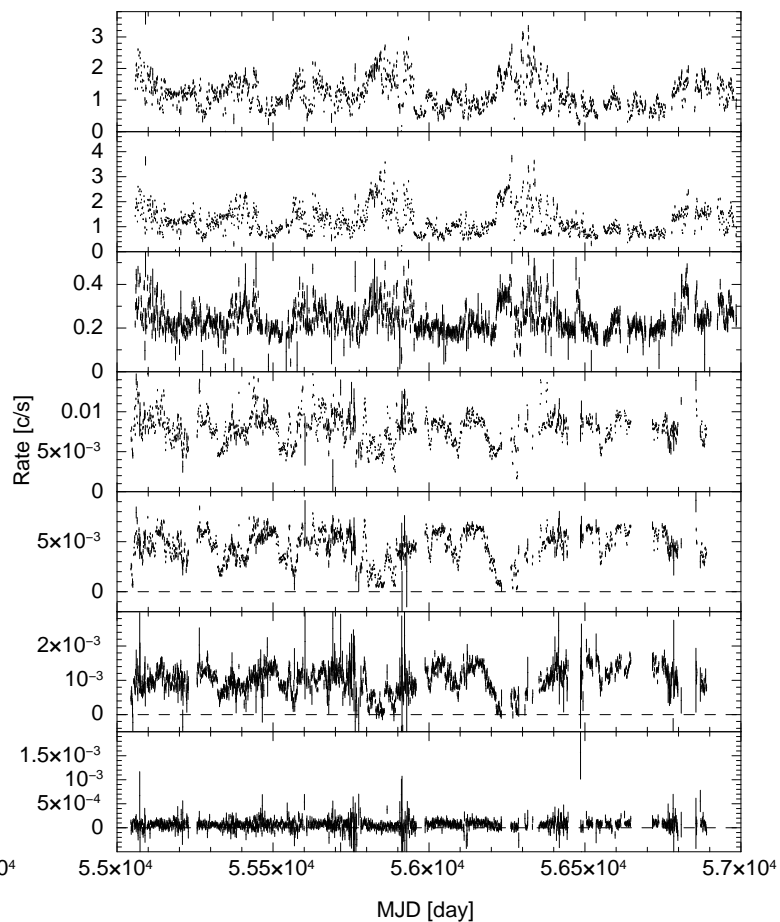

Figure 4: GRS 1915+105 


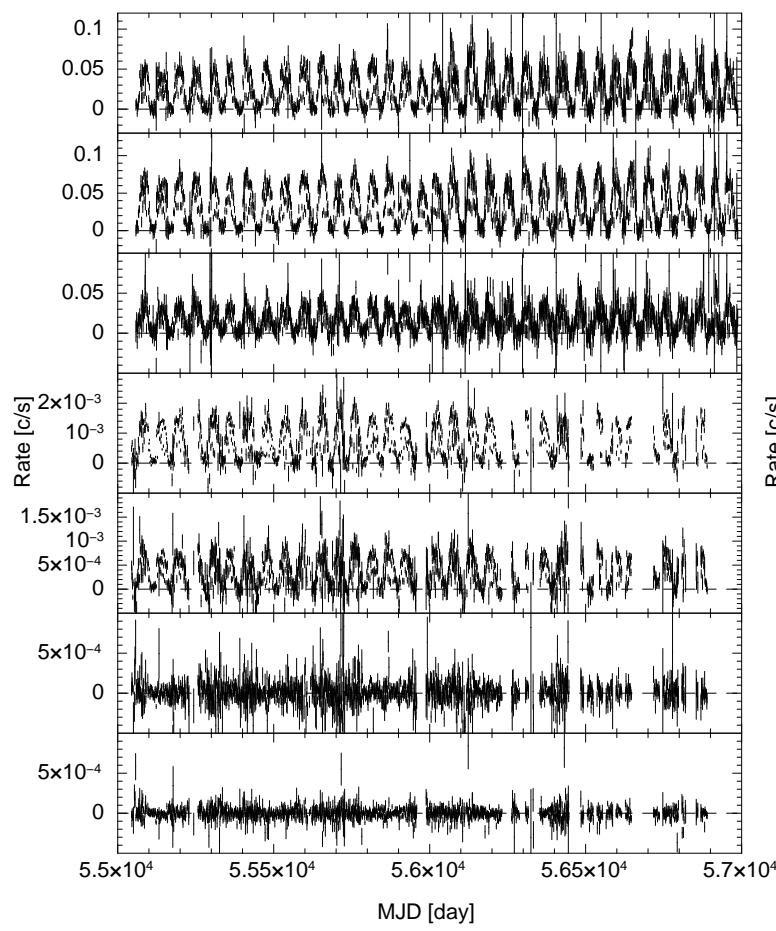

Figure 5: SMC X-1

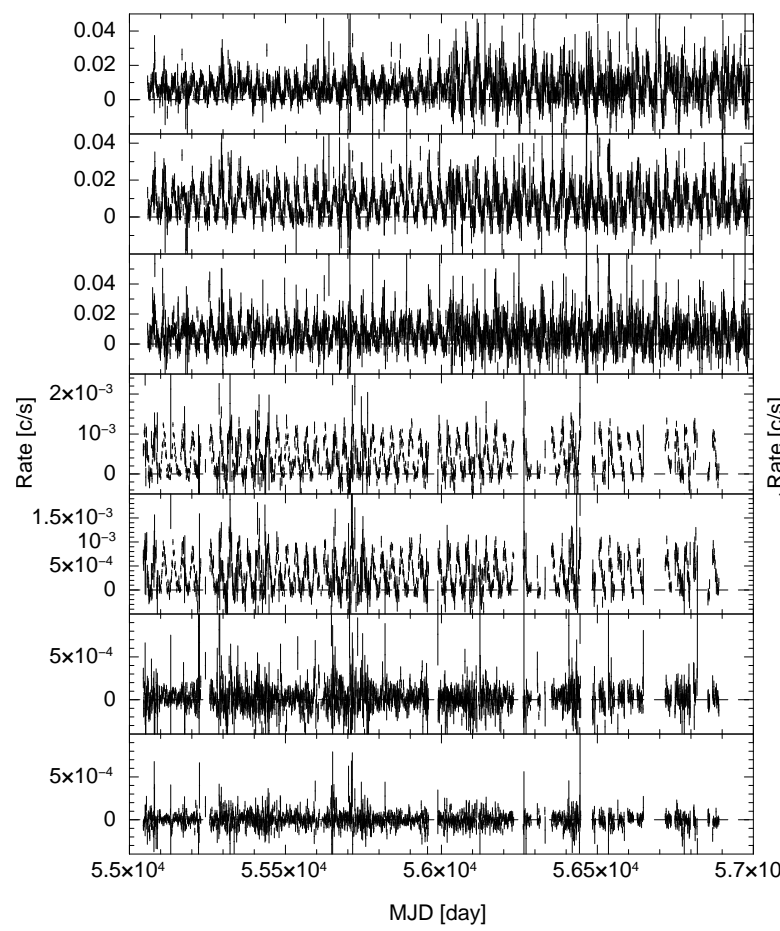

Figure 7: LMC X-4

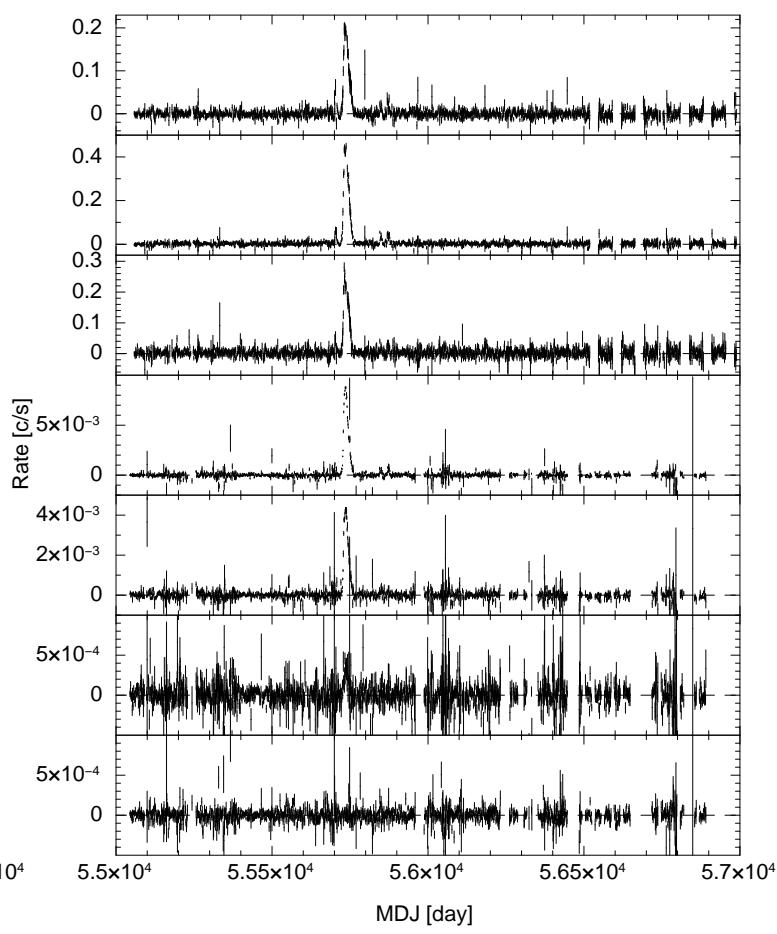

Figure 6: 4U 0115+634

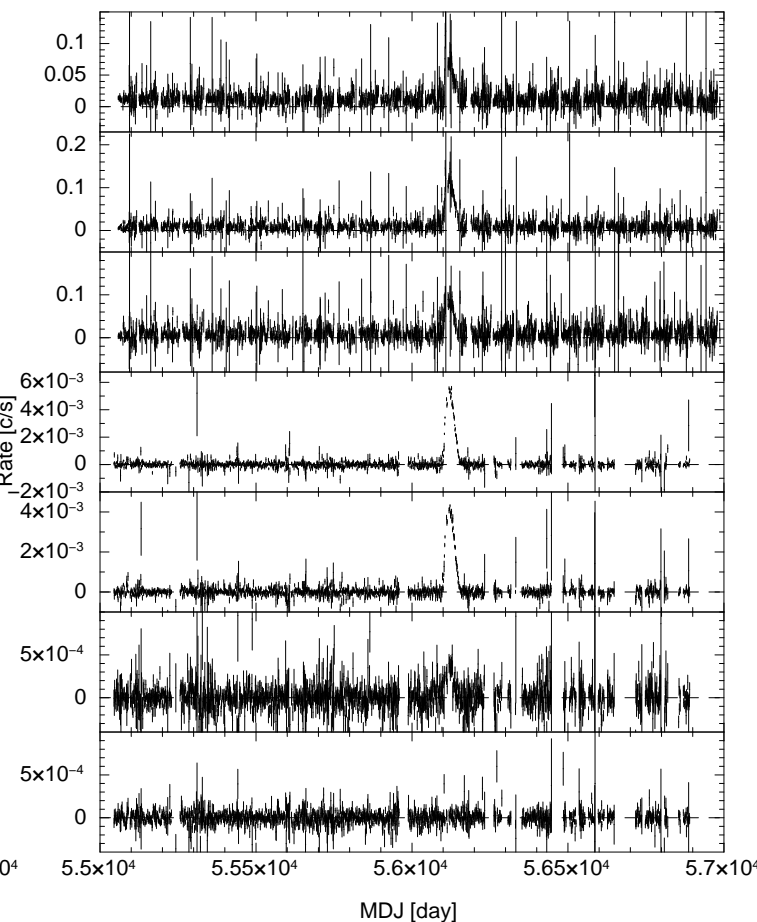

Figure 8: GS 0834-430 